\title{
Geometric Study of a Family of Integrable Systems
}

\author{
Ahmed Lesfari* \\ (Communicated by Kazım İlarslan)
}

\begin{abstract}
The aim of this paper is to demonstrate the rich interaction between complex algebraic geometry, the theory of integrable systems and the geometry of its asymptotic solutions. We present a family of integrable hamiltonian systems. We study theses systems from a different angle, assemble different geometric methods and several views.
\end{abstract}

Keywords: Integrable systems; gap theory; abelian varieties; jacobian varieties; embeddings.

AMS Subject Classification (2010): Primary: 70H06; Secondary: 14H40; 14H70; 14E25; 32Q15.

\section{Introduction}

In this paper, we shall be concerned with finite dimensional algebraic completely integrable systems. A dynamical system is algebraic completely integrable $[2,3,30]$ if it can be linearized on an abelian variety; a complex algebraic torus $\mathbb{C}^{n} /$ lattice. The invariants (often called first integrals or constants) of the motion are polynomials and the phase space coordinates (or some algebraic functions of these) restricted to a complex invariant variety defined by putting these invariants equals to generic constants, are meromorphic functions on an abelian variety. Moreover, in the coordinates of this abelian variety, the flows (run with complex time) generated by the constants of the motion are straight lines. However, besides the fact that many hamiltonian completely integrable systems posses this structure, another motivation for its study is algebraic completely integrable systems come up systematically whenever you study the isospectral deformation of some linear operator containing a rational indeterminate. Indeed a theorem of Adler-Kostant-Symes [3] applied to KacMoody algebras provides such systems which, by a theorem of van Moerbeke-Mumford [44], are algebraic completely integrable. Therefore there are hidden symmetries which have a group theoretical foundation. In recent decades there has been much effort given for finding integrable hamiltonian systems, not only because they have been on the subject of powerful and beautiful theories of mathematics, but also because the concepts of integrability have been applied to an increasing number of physical systems, biological phenomena, population dynamics, chemical rate equations, to mention only a few. However, it seems still hopeless to describe or even to recognize with any facility, those hamiltonian systems which are integrable, though they are quite exceptional. In fact, the overwhelming majority of dynamical systems, hamiltonian or not, are non-integrable and possess regimes of chaotic behavior in phase space. The concept of algebraic complete integrability is quite effective in small dimensions and has the advantage to lead to global results, unlike the existing criteria for real analytic integrability, which, at this stage are perturbation results.

In the present paper, we discuss an interesting interaction between complex algebraic geometry and dynamics. The paper is organized into eight sections, the first of which is this introduction. In section 2, we discuss the integrability of a family of hamiltonian systems. In section 3, we study this problem using the Lax spectral curve technique. We show that the system (2.2) in this case is linearized in the jacobian variety of a genus two hyperelliptic Riemann surface $\Gamma$. In section 4 , we solve the equations of motion in terms of elliptic functions with the help of finite-gap integration theory and this can be done only for some special relations between the parameters $a_{1}, a_{2}$ and the constants of motion. The main problem of sections 5,6 and 7 , is to show that the affine surface $V_{c}$ (3.5) obtained by setting the two quartics invariants of the problem equal to generic 
constants, is the affine part of an abelian surface $\widetilde{V}_{c}$. A naive guess would be to take the natural completion $\bar{V}_{c}$ of $V_{c}$ in $\mathbb{C P}^{4}$. We show that $\bar{V}_{c}$ is not an abelian surface and $\bar{V}_{c}$ must have a singularity somewhere along the locus at infinity $I=\bar{V}_{c} \cap\left\{X_{0}=0\right\}$, the latter consists of two lines $d_{\varepsilon}, \varepsilon= \pm 1$. The trajectories of the vectors fields (5.2) hit every point of these lines. We prove that around the points of hitting, the system of differential equations (5.2) admit Laurent expansion solutions whose coefficients depend on three free parameters. These parameters can be used to blowing up the variety $\bar{V}_{c}$ along the lines $d_{\varepsilon}(\varepsilon= \pm 1)$. The new complex surface obtained is an abelian surface $\widetilde{V}_{c}$ and the two lines $d_{\varepsilon}$ turn into two copies $C_{\varepsilon}(\varepsilon= \pm 1)$ of the same smooth genus 3 hyperelliptic curve $C$, intersecting in two distinct points at which they are tangent to each other. The curve $C$ is a double ramified cover of an elliptic curve $C_{0}$ ramified at four points and a $2-1$ unramified cover of a genus 2 hyperelliptic curve $\mathcal{H}$. The 16 functions $1, f_{1}, \ldots, f_{15}$ defined in (7.4) provide the embedding of $\widetilde{V}_{c}$ in projective 15 -space $\mathbb{C P}^{15}$. The divisor $D=C_{+1}+C_{-1}$ obtained in this way has genus 5 and thus $S=2 D$ has genus 17. In section 7 , we prove that the flow (5.3) evolves on abelian surface $\widetilde{V}_{c}=\mathbb{T}_{c}^{2} \subset \mathbb{C P}^{15}$ with the polarization of type $(2,2)$ and it will be expressed in terms of abelian integrals, involving the differentials (7.5). In section 8 , we present a brief discussion and concluding remarks about similar problems.

\section{A family of integrable systems}

We consider the hamiltonian

$$
H=\frac{1}{2}\left(x_{1}^{2}+x_{2}^{2}+a_{1} y_{1}^{2}+a_{2} y_{2}^{2}\right)+\frac{1}{4} y_{1}^{4}+\frac{1}{4} a_{3} y_{2}^{4}+\frac{1}{2} a_{4} y_{1}^{2} y_{2}^{2},
$$

where $a_{1}, a_{2}, a_{3}, a_{4}$ are arbitrary constants. The corresponding system is given by

$$
\ddot{y}_{1}+\left(a_{1}+y_{1}^{2}+a_{4} y_{2}^{2}\right) y_{1}=0, \quad \ddot{y}_{2}+\left(a_{2}+a_{3} y_{2}^{2}+a_{4} y_{1}^{2}\right) y_{2}=0 .
$$

The integrability of this hamiltonian system has been studied by several authors. It arises in connection with some problems in scalar field theory and in the semi-classical method in quantum field theory. It corresponds to the Garnier system and to the anisotropic harmonic oscillator in a radial quartic potential. Also, this hamiltonian can be connected to the coupled nonlinear Schrödinger equations. For more information on these connections, noticed long ago, we can see for example the following articles : [11, 12, 24, 38, 46, 48 and 50]. Furthermore, the hamiltonian (2.1) is part of the well known family

$$
H=\frac{1}{2}\left(x_{1}^{2}+x_{2}^{2}\right)+\frac{1}{2}\left(a_{1} y_{1}^{2}+a_{2} y_{2}^{2}\right)+A y_{1}^{4}+B y_{1}^{2} y_{2}^{2}+C y_{2}^{4}+\frac{1}{2}\left(\frac{E}{y_{1}^{2}}+\frac{D}{y_{2}^{2}}\right),
$$

which describe the motion of particles interacting with a quartic potential $A y_{1}^{4}+B y_{1}^{2} y_{2}^{2}+C y_{2}^{4}$ and perturbed by an inverse squared potential. There are four nontrivial known cases of complete integrability for the nonperturbed quartic potential and were all studied in detail from different points of view: 1) A:B:C=1:2:1, 2) $\mathrm{A}: \mathrm{B}: \mathrm{C}=1: 12: 16$, 3) $\mathrm{A}: \mathrm{B}: \mathrm{C}=1: 6: 1$, 4) $\mathrm{A}: \mathrm{B}: \mathrm{C}=1: 6: 8$. Cases 1), 2) and 3) are separable in ellipsoidal, parabolical and cartesian coordinates respectively, whilst case 4) is separable in the general sense [39]. The integrability of case (i) and separability in ellipsoidal coordinates was proved in [47] (see also [25, 41]). The case 2) appears as one of the entries in the polynomial hierarchy discussed in [14]. The cases 3) and 4) are proved to be canonically equivalent under the action of a Miura map restricted to the stationary coupled KdV systems associated with a fourth order Lax operator [6]. Moreover all the cases 1)-4) allow the deformation of the potential by linear combination of inverse squares and squares with certain limitations on the coefficients (see [11], [4]). There are also Lax representations known for all these cases which yield hyperelliptic algebraic curves in the cases 1) and 2) and a 4-gonal curve in the cases 3) and 4). Various results concerning cases 1)-4) can be found in $[22,23,17,38]$. For a review of known results see also [13, 45] and the references therein. When $a_{1}=a_{2}=A=C=E=D=0, B=\frac{1}{2}$, this hamiltonian can be connected to the Yang-Mills system for a field with gauge group $S U(2)$ (indeed, consider the Yang-Mills system : $D_{i} F_{i k}=\partial_{i} F_{i k}+\left[A_{i}, F_{i k}\right]=0$, where $F_{i k}, A_{i} \in$ $T_{e} S U(2), 1 \leq i, k \leq 4$ and $F_{i k}=\partial_{i} A_{k}-\partial_{k} A_{i}+\left[A_{i}, A_{k}\right]$. In the case of homogeneous two-component field, we have $\partial_{i} A_{k}=0, i \neq 1, A_{1}=A_{2}=0, A_{3}=n_{1} U_{1}, A_{4}=n_{2} U_{2}, n_{1,2}=$ constant, $n_{1}=\left[n_{2},\left[n_{1}, n_{2}\right]\right], n_{2}=\left[n_{1},\left[n_{2}, n_{1}\right]\right]$ and the system becomes $\partial^{2} U_{1}+U_{1} U_{2}^{2}=0, \partial^{2} U_{2}+U_{2} U_{1}^{2}=0$. By setting $U_{1}=y_{1}, U_{2}=y_{2}, \frac{\partial U_{1}}{\partial t}=x_{1}, \frac{\partial U_{2}}{\partial t}=x_{2}$, Yang-Mills equations are reduced to hamiltonian system with the hamiltonian $H=\frac{1}{2}\left(x_{1}^{2}+x_{2}^{2}+y_{1}^{2} y_{2}^{2}\right)$. The self-dual Yang-Mills (SDYM) equations is an universal system for which some reductions include all classical 
tops from Euler to Kowalewski (0+1-dimensions), K-dV, Nonlinear Schrödinger, Sine-Gordon, Toda lattice and $\mathrm{N}$-waves equations (1+1-dimensions), KP and D-S equations (2+1-dimensions).

The hamiltonian (2.1) has the Painlevé property (i.e. the general solutions have no movable singularities other than poles) only if $i$ ) $\left.a_{1}=a_{2}, a_{3}=a_{4}=1, i i\right) a_{1}=a_{2}, a_{3}=1, a_{4}=3$. In the case $i$ ), the second integral has the form $H_{2}=x_{2} y_{1}-x_{1} y_{2}$, whereas in the case $\left.i i\right)$ the second integral is $H_{2}=x_{1} x_{2}+y_{1} y_{2}\left(a_{1}+y_{1}^{2}+y_{2}^{2}\right)$. Also, it was shown [24] that if $i$ ii) $a_{1}=\frac{a_{2}}{4}, a_{3}=16, a_{4}=6$, the system (2.2) is integrable and the second integral is $H_{2}=a_{1} y_{1}^{2}+y_{1} y_{2}\left(y_{1}^{3}+2 y_{1} y_{2}^{2}\right)+x_{1}\left(x_{2} y_{1}-x_{1} y_{2}\right)$. This paper deals with the problem of integrability of the system (2.2) corresponding to the choice : $a_{1}, a_{2}$ arbitrary and $a_{3}=a_{4}=1$. For $a_{1}=a_{2}$, it is easy to show that the problem can be integrated in terms of elliptic functions. In the next sections, we suppose that $a_{1} \neq a_{2}$.

\section{Lax representation and integrability}

Using the results given in $[14,15]$, we consider the Lax representation in the form

$$
\dot{A}_{h}=\left[B_{h}, A_{h}\right]=B_{h} A_{h}-A_{h} B_{h}, \quad \cdot \equiv \frac{\partial}{\partial t}
$$

with the following ansatz for the Lax operator

$$
A_{h}=\left(\begin{array}{cc}
u(h) & v(h) \\
w(h) & -u(h)
\end{array}\right), \quad B_{h}=\left(\begin{array}{cc}
0 & 1 \\
r(h) & 0
\end{array}\right),
$$

where

$$
\begin{aligned}
v(h) & =-\left(a_{1}+h\right)\left(a_{2}+h\right)\left(1+\frac{1}{2}\left(\frac{y_{1}^{2}}{a_{1}+h}+\frac{y_{2}^{2}}{a_{2}+h}\right)\right), \\
u(h) & =\frac{1}{2}\left(a_{1}+h\right)\left(a_{2}+h\right)\left(\frac{x_{1} y_{1}}{a_{1}+h}+\frac{x_{2} y_{2}}{a_{2}+h}\right), \\
w(h) & =\left(a_{1}+h\right)\left(a_{2}+h\right)\left(\frac{1}{2}\left(\frac{x_{1}^{2}}{a_{1}+h}+\frac{x_{2}^{2}}{a_{2}+h}\right)-h+\frac{1}{2}\left(y_{1}^{2}+y_{2}^{2}\right)\right), \\
r(h) & =h-y_{1}^{2}-y_{2}^{2} .
\end{aligned}
$$

The system (2.2) where $a_{3}=a_{4}=1$ admits a Lax representation given by (3.1). The proof is straightforward and based on direct computation : we have

$$
\left[B_{h}, A_{h}\right]=\left(\begin{array}{cc}
w(h)-v(h) r(h) & -2 u(h) \\
2 u(h) r(h) & v(h) r(h)-w(h)
\end{array}\right),
$$

and it follows from (3.1), (3.2) and (2.2) with $a_{3}=a_{4}=1$ that

$$
\dot{u}(h)=w(h)-v(h) r(h), \quad \dot{v}(h)=-2 u(h), \quad \dot{w}(h)=2 u(h) r(h) .
$$

Equation (3.1) means that for $h \in \mathbb{C}$ and under the time evolution of the system, $A_{h}(t)$ remain similar to $A_{h}(0)$. So the spectrum of $A_{h}$ is conserved, i.e. it undergoes an isospectral deformation. The eigenvalues of $A_{h}$, viewed as functionals, represent the integrals (constants of the motion) of the system. To be precise, a hamiltonian flow of the type (3.1) preserves the spectrum of $A_{h}$ and therefore its characteristic polynomial $\operatorname{det}\left(A_{h}-z I\right)$. We form the Riemann surface in $(z, h)$ space $: \Gamma: \operatorname{det}\left(A_{h}-z I\right)=0$, whose coefficients are functions of the phase space. Explicitly, this equation looks as follows

$$
\begin{aligned}
\Gamma: z^{2} & =u^{2}(h)+v(h) w(h) \equiv P_{5}(h), \\
& =\left(a_{1}+h\right)\left(a_{2}+h\right)\left(h^{3}+\left(a_{1}+a_{2}\right) h^{2}+\left(a_{1} a_{2}-H_{1}\right) h-H_{2}\right),
\end{aligned}
$$

where $H_{1}=H$ is defined by (2.1) with $a_{1}, a_{2}$ arbitrary, $a_{3}=a_{4}=1$ and a second quartic integral $H_{2}$ of the form

$$
\begin{aligned}
H_{2}= & \frac{1}{4}\left(a_{2} y_{1}^{4}+a_{1} y_{2}^{4}+\left(a_{1}+a_{2}\right) y_{1}^{2} y_{2}^{2}+\left(x_{1} y_{2}-x_{2} y_{1}\right)^{2}\right) \\
& +\frac{1}{2}\left(a_{2} x_{1}^{2}+a_{1} x_{2}^{2}+a_{1} a_{2}\left(y_{1}^{2}+y_{2}^{2}\right)\right) .
\end{aligned}
$$


The curve $\Gamma$ determined by the fifth-order equation (3.3) is smooth, hyperelliptic and its genus is two. Obviously, $\Gamma$ is invariant under the involution $(h, z) \longmapsto(h,-z)$. The second hamiltonian vector field is written as

$$
\begin{aligned}
& \dot{y}_{1}=\frac{1}{2}\left(x_{1} y_{2}-x_{2} y_{1}\right) y_{2}+a_{2} x_{1}, \quad \dot{y}_{2}=-\frac{1}{2}\left(x_{1} y_{2}-x_{2} y_{1}\right) y_{1}+a_{1} x_{2}, \\
& \dot{x}_{1}=-a_{2} y_{1}^{3}-\frac{1}{2}\left(a_{1}+a_{2}\right) y_{1} y_{2}^{2}+\frac{1}{2}\left(x_{1} y_{2}-x_{2} y_{1}\right) x_{2}-a_{1} a_{2} y_{1}, \\
& \dot{x}_{2}=-a_{1} y_{2}^{3}-\frac{1}{2}\left(a_{1}+a_{2}\right) y_{1}^{2} y_{2}-\frac{1}{2}\left(x_{1} y_{2}-x_{2} y_{1}\right) x_{1}-a_{1} a_{2} y_{2} .
\end{aligned}
$$

These vector fields are in involution with respect to the associated Poisson bracket. For generic $c=\left(c_{1}, c_{2}\right) \in \mathbb{C}^{2}$,

$$
V_{c}=\left\{H_{1}=c_{1}, H_{2}=c_{2}\right\},
$$

is a smooth affine surface. The linearized flow could be realized on the jacobian variety $\operatorname{Jac}(\Gamma)$ of the curve (3.3). Indeed, as the structure of the matrices $A_{h}$ and $B_{h}$ (degree of the polynomials in $h$ ) is the same as for the well-known Neumann system, so both systems are linearized in the same way. We can construct an algebraic map from $V_{c}$ to the Jacobi variety $J a c(\Gamma)$ :

$$
V_{c} \longrightarrow J a c(\Gamma), \quad p \in V_{c} \longmapsto\left(s_{1}+s_{2}\right) \in \operatorname{Jac}(\Gamma),
$$

and the flows generated by the constants of the motion are straight lines on $J a c(\Gamma)$, i.e., the linearizing equations are given by

$$
\sum_{i=1}^{2} \int_{s_{i}(0)}^{s_{i}(t)} \omega_{k}=c_{k} t, \quad 0 \leq k \leq 2
$$

where $\omega_{1}, \omega_{2}$ span the two-dimensional space of holomorphic differentials on the curve $\Gamma$ and $s_{1}, s_{2}$ two appropriate variables, algebraically related to the originally given ones, for which the Hamilton-Jacobi equation could be solved by separation of variables. Consequently, we have

Theorem 3.1. The system (2.2) is completely integrable for all $a_{1}, a_{2}, a_{3}=a_{4}=1$ and admits a Lax representation given by (3.1). The invariants of $A_{h}$ are integrals of motion in involution. The first integral is given by the hamiltonian $H_{1}=H$ (2.1) whereas the second integral $H_{2}$ is also quartic and has the form (3.4). The flows generated by $H_{1}$ and $H_{2}$ are straight line motions on the jacobian variety $J a c(\Gamma)$ of a smooth genus two hyperelliptic curve $\Gamma$ (3.3) associated to Lax equation (3.1).

We introduce coordinates $s_{1}$ and $s_{2}$ on the surface $V_{c}(3.5)$, such that $v\left(s_{1}\right)=v\left(s_{2}\right)=0, a_{1} \neq a_{2}$,

$$
y_{1}^{2}=2 \frac{\left(a_{1}+s_{1}\right)\left(a_{1}+s_{2}\right)}{a_{1}-a_{2}}, \quad y_{2}^{2}=2 \frac{\left(a_{2}+s_{1}\right)\left(a_{2}+s_{2}\right)}{a_{2}-a_{1}},
$$

i.e.,

$$
s_{1}+s_{2}=\frac{1}{2}\left(y_{1}^{2}+y_{2}^{2}\right)-a_{1}-a_{2}, \quad s_{1} s_{2}=-\frac{1}{2}\left(a_{2} y_{1}^{2}+a_{1} y_{2}^{2}\right)+a_{1} a_{2} .
$$

After some manipulations, we obtain the following equations for $s_{1}$ and $s_{2}$ :

$$
\dot{s}_{1}=2 \frac{\sqrt{P_{5}\left(s_{1}\right)}}{s_{1}-s_{2}}, \quad \dot{s}_{2}=2 \frac{\sqrt{P_{5}\left(s_{2}\right)}}{s_{2}-s_{1}},
$$

where $P_{5}(s)$ is defined by (3.3). These equations can be integrated by the abelian mapping

$$
\Gamma \longrightarrow \operatorname{Jac}(\Gamma)=\mathbb{C}^{2} / L, \quad p \longmapsto\left(\int_{p_{0}}^{p} \omega_{1}, \int_{p_{0}}^{p} \omega_{2}\right),
$$

where the genus two hyperelliptic curve $\Gamma$ is given by the equation (3.3), $L$ is the lattice generated by the vectors $n_{1}+\Omega n_{2},\left(n_{1}, n_{2}\right) \in \mathbb{Z}^{2}, \Omega$ is the matrix of period of $\Gamma,\left(\omega_{1}, \omega_{2}\right)$ is a basis of holomorphic differentials on $\Gamma$, i.e.,

$$
\omega_{1}=\frac{d s}{\sqrt{P_{5}(s)}}, \quad \omega_{2}=\frac{s d s}{\sqrt{P_{5}(s)}},
$$

and $p_{0}$ is a fixed point. Consequently, we have

Theorem 3.2. The system of differential equations (2.2) with $a_{3}=a_{4}=1$, can be integrated in terms of genus two hyperelliptic functions of time. 


\section{The gap theory and linearization using elliptic functions}

In this section, we show that at some special values of the parameters $a_{1}$ and $a_{2}$, the solutions for the integrable case proposed in this paper may be expressed in terms of elliptic functions. We solve the equations of motion with the help of finite-gap integration theory. The reader is referred to the book [10] for this theory and its applications to nonlinear integrable equations. For doing that, we need some notions about elliptic Weierstrass $\wp$-function which we summarize here after.

Let $\Lambda=\left\{2 m \omega_{1}+2 n \omega_{2},(m, n) \in \mathbb{Z}^{2}\right\} \subset \mathbb{C}$ be a lattice. We recall that the elliptic Weierstrass $\wp$-function with respect to $\Lambda$ is defined by

$$
\wp(z)=\frac{1}{z^{2}}+\sum_{\substack{(m, n) \neq(0,0) \\(m, n) \in \mathbb{Z}^{2}}}\left\{\frac{1}{\left(z-2 m \omega_{1}-2 n \omega_{2}\right)^{2}}-\frac{1}{\left(2 m \omega_{1}+2 n \omega_{2}\right)^{2}}\right\}, z \in \mathbb{C} .
$$

This series converges absolutely and uniformly on every compact set in $\mathbb{C} \backslash \Lambda$, thus giving a meromorphic function having a double pole at each lattice point. This function is doubly periodic $\wp\left(z+2 m \omega_{1}+2 n \omega_{2}\right)=\wp(z)$, $(m, n \in \mathbb{Z})$ with periods $2 \omega_{1}, 2 \omega_{2}$ such that $\operatorname{Im} \frac{\omega_{2}}{\omega_{1}}>0$ and has a singularity at $z=0$ such as

$$
\begin{gathered}
\wp(z)=\frac{1}{z^{2}}+\frac{g_{2}}{20} z^{2}+\frac{g_{3}}{28} z^{4}+\frac{g_{2}^{2}}{1200} z^{6}+\cdots, \\
g_{2}=60 \sum_{\substack{(m, n) \neq(0,0) \\
(m, n) \in \mathbb{Z}^{2}}} \frac{1}{\left(2 m \omega_{1}+2 n \omega_{2}\right)^{2}}, \quad g_{3}=140 \sum_{\substack{(m, n) \neq(0,0) \\
(m, n) \in \mathbb{Z}^{2}}} \frac{1}{\left(2 m \omega_{1}+2 n \omega_{2}\right)^{6}} .
\end{gathered}
$$

The derivative $\wp^{\prime}(z)$ is again an elliptic function. The $\wp$-function satisfies the addition theorem

$$
\operatorname{det}\left(\begin{array}{ccc}
1 & \wp(a) & \wp^{\prime}(a) \\
1 & \wp(b) & \wp^{\prime}(b) \\
1 & \wp(c) & \wp^{\prime}(c)
\end{array}\right)=0, \quad a+b+c=0
$$

as well as the differential equation

$$
\begin{aligned}
\left(\wp^{\prime}(z)\right)^{2} & =4\left(\wp(z)-e_{1}\right)\left(\wp(z)-e_{2}\right)\left(\wp(z)-e_{3}\right) \\
& =4 \wp^{3}(z)-g_{2} \wp(z)-g_{3}
\end{aligned}
$$

where $e_{1}=\wp\left(\omega_{1}\right), e_{2}=\wp\left(\omega_{1}+\omega_{2}\right)$ and $e_{3}=\wp\left(\omega_{2}\right)$. The map

$$
\mathbb{C} / \Lambda \longrightarrow \mathbb{C P}^{2}, z \longmapsto\left[1, \wp(z), \wp^{\prime}(z)\right], z \neq 0 \text { and } 0 \longmapsto[0,0,1],
$$

is an isomorphism between the complex torus $\mathbb{C} / \Lambda$ and the elliptic curve

$$
y^{2}=4 x^{3}-g_{2} x-g_{3},
$$

where $x=\wp(z)$ and $y=\wp^{\prime}(z)$.

The elliptic solutions for which we look for are given by the spectral problem (the Schrödinger equation) :

$$
\left(\frac{\partial^{2}}{\partial x^{2}}-\mathcal{U}(x)\right) \Psi=\lambda \Psi
$$

depending in the elliptic potential

$$
\mathcal{U}(x)=2 \sum_{i=1}^{3} \wp\left(x-x_{i}\right)+C .
$$

Here $\Psi=\Psi(x, \lambda)$ is an eigenfunction (elliptic Backer-Akhiezer function) of operator $\mathcal{L}, \wp$ is the elliptic Weierstrass function defined above, $C$ is a constant and $x_{1}, x_{2}, x_{3}$ are on the locus

$$
\Delta=\left\{\left(x_{1}, x_{2}, x_{3}\right) \in \mathbb{C}^{3}: \sum_{i \neq j} \wp^{\prime}\left(x_{i}-x_{j}\right)=0, x_{i} \neq x_{j}, j=1,2,3\right\} .
$$


The locus $\Delta$ is non-empty and its geometry was studied by several authors [4, 32]. It is shown in [4] that if $x_{i}=x_{i}(t), j=1,2,3$ evolve according to the law

$$
\dot{x}_{i}=-12 \sum_{i \neq j} \wp\left(x_{i}-x_{j}\right)
$$

then the function (4.2) is an elliptic solution of the well-known Korteweg-de Vries equation (KdV) :

$$
\frac{\partial u}{\partial t}-6 u \frac{\partial u}{\partial x}+\frac{\partial^{3} u}{\partial x^{3}}=0
$$

and is connected with the completely integrable Calogero-Moser system described by the hamiltonian :

$$
H=\frac{1}{2} \sum_{i=1}^{3} y_{i}^{2}-2 \sum_{i \neq j} \wp\left(x_{i}-x_{j}\right)
$$

with $y_{i}, x_{i}, i=1,2,3$ being canonical variables. The Jacobi inversion problem associated with 2-gap solutions of the $\mathrm{KdV}$ equation is determined by

$$
\int_{\infty}^{s_{1}} \frac{d z}{w}+\int_{\infty}^{s_{2}} \frac{d z}{w}=-8 i t+c_{1}, \quad \int_{\infty}^{s_{1}} \frac{z d z}{w}+\int_{\infty}^{s_{2}} \frac{z d z}{w}=2 i x+c_{2},
$$

where $c_{1}, c_{2}$ are constants, $w^{2}=\prod_{j=1}^{5}\left(z-z_{j}\right), s_{j}=s_{j}(x, t), j=1,2$ and $z_{j}, j=1, \ldots, 5$, are expressed in terms of integrals of motion. After an appropriate change of variables and corresponding choices of $z_{j}$, the Jacobi inversion problem above coincides with that associated with Hénon-Heiles equations [16]. Let us consider the two-gap potential for equation (4.2) normalized by its expansion near $x=0$ as

$$
\mathcal{U}(x)=\frac{6}{x^{2}}+\alpha_{1} x^{2}+\alpha_{2} x^{4}+\alpha_{3} x^{6}+\alpha_{4} x^{8}+O\left(x^{10}\right)
$$

where $\alpha_{1}, \alpha_{2}, \alpha_{3}, \alpha_{4}$ are functions of the moduli $g_{2}, g_{3}$ of the underlying elliptic curve. This potential satisfies the Novikov equation [37]: $\sum_{i=-1}^{2} a_{i} \frac{\delta S_{i}}{\delta u}=0$, where $\delta$ is the variational operator of the calculus of variations, $a_{1}, a_{2}$, are constants and

$$
\begin{gathered}
S_{-1}=\int u d x, \quad S_{1}=\int\left(\frac{1}{2}\left(\frac{\partial u}{\partial x}\right)^{2}+u^{3}\right) d x \\
S_{0}=\int u^{2} d x, \quad S_{2}=\int\left(\frac{1}{2}\left(\frac{\partial^{2} u}{\partial x^{2}}\right)^{2}-\frac{5}{2} u^{2} \frac{\partial^{2} u}{\partial x^{2}}+\frac{5}{2} u^{4}\right) d x,
\end{gathered}
$$

are the first integrals of the KdV equation. The algebraic curve associated with the potential (4.3) has the form [9]:

$$
w^{2}=z^{5}-\frac{35}{2} \alpha_{1} z^{3}-\frac{63}{2} \alpha_{2} z^{2}+\frac{1}{4}\left(\frac{567}{2} \alpha_{1}^{2}+297 \alpha_{3}\right) z+\frac{1377}{4} \alpha_{1} \alpha_{2}-\frac{1287}{2} \alpha_{4} .
$$

As the potential is elliptic (2-gap Lamé potential) the curve (4.4) can be written explicitly as, $w^{2}=\left(z^{2}-3 g_{2}\right)(z-$ $\left.3 e_{1}\right)\left(z-3 e_{2}\right)\left(z-3 e_{3}\right)$. All this can be done by classical means following the work of Hermite [21] and Halphen [20]. In addition, it follows from the trace formulas [49] that

$$
\begin{gathered}
s_{1}+s_{2}=-\sum_{j=1}^{3} \wp\left(x-x_{j}\right)+\frac{1}{2} \sum_{j=1}^{5} z_{j}-\frac{C}{2} \\
s_{1} s_{2}=3 \sum_{\substack{i, j=1 \\
i\langle j}}^{3} \wp\left(x-x_{i}\right) \wp\left(x-x_{j}\right)-\frac{3}{8} g_{2}+\frac{1}{2} \sum_{\substack{i, j=1 \\
i\langle j}}^{5} z_{i} z_{j}-\frac{3}{8}\left(\sum_{j=1}^{5} z_{j}\right)^{2} \\
+\frac{3 C}{2} \sum_{j=1}^{3} \wp\left(x-x_{j}\right)+\frac{3 C^{2}}{8},
\end{gathered}
$$


where $x_{1}, x_{2}, x_{3} \in \Delta$ and $z_{1}, \ldots, z_{5}$ are the branching points of the genus 2 hyperelliptic curve (4.4). We want the curve $\Gamma$ (3.3) to be associated with the genus 2 curve (4.4). Let $z_{\alpha}, z_{\beta}$ be two distinct branch points of the curve (4.4) which are the shifted values of the branch points $a_{1} a_{2}$ of the curve $\Gamma$ (3.3). From system (2.2), we obtain

$$
\ddot{y}_{1}=\left(a_{1}-y_{1}^{2}-y_{2}^{2}\right) y_{1}, \quad \ddot{y}_{2}=\left(a_{2}-y_{1}^{2}-y_{2}^{2}\right) y_{2},
$$

implying that

$$
\ddot{y}_{1}-\mathcal{U}(x) y_{1}=\left(a_{1}-2\left(z_{\alpha}+y_{\beta}\right)\right) y_{1}, \quad \ddot{y}_{2}-\mathcal{U}(x) y_{2}=\left(a_{2}-2\left(z_{\alpha}+y_{\beta}\right)\right) y_{2} .
$$

Consequently, we have

Theorem 4.1. Suppose that $a_{1}=3 z_{\alpha}+2 z_{\beta}$ and $a_{2}=2 z_{\alpha}+3 z_{\beta}$ where $z_{\alpha}$ and $z_{\beta}$ are two distinct branch points of the curve (4.4). Then, formula for elliptic solutions of (2.2) is given by

$$
\begin{aligned}
& y_{1}^{2}=\frac{1}{z_{\alpha \beta}}\left\{\Phi+2 z_{\alpha}^{2}+\left(3 C-2 z_{\alpha}\right) \sum_{i=1}^{3} \wp\left(x-x_{i}\right)+\left(\frac{3 C}{4}-z_{\alpha}\right) C\right\}, \\
& y_{2}^{2}=\frac{-1}{z_{\alpha \beta}}\left\{\Phi+2 z_{\beta}^{2}+\left(3 C-2 z_{\beta}\right) \sum_{i=1}^{3} \wp\left(x-x_{i}\right)+\left(\frac{3 C}{4}-z_{\beta}\right) C\right\},
\end{aligned}
$$

where $z_{\alpha \beta} \equiv z_{\alpha}-z_{\beta}, \Phi \equiv 6 \sum_{1 \leq i\langle j \leq 3} \wp\left(x-x_{i}\right) \wp\left(x-x_{j}\right)-\frac{3 g_{2}}{4}+\sum_{1 \leq i<j \leq 5} z_{i} z_{j}$ and $z_{1}, \ldots, z_{5}$ are the branching points of the curve (2.2).

\section{Algebraic complete integrability and intersection of quartics in complex projective 4-space}

First we recall some basics concepts. Consider hamiltonian problems of the form

$$
\dot{x}=J \frac{\partial H}{\partial x}, x \in \mathbb{R}^{m},
$$

where $H$ is the hamiltonian and $J=J(x)$ is a skew-symmetric matrix with polynomial entries in $x$, for which the corresponding Poisson bracket $\left\{H_{i}, H_{j}\right\}=\left\langle\frac{\partial H_{i}}{\partial x}, J \frac{\partial H_{j}}{\partial x}\right\rangle$, satisfies the Jacobi identity. The system (5.1) with polynomial right hand side is called algebraic complete integrable when :

a) The system (5.1) possesses $n+k$ independent polynomial invariants $H_{1}, \ldots, H_{n+k}$ of which $k$ lead to zero vector fields $J \frac{\partial H_{n+i}}{\partial x}=0,1 \leq i \leq k$ (called Casimir functions) and the $n$ remaining ones are in involution (i.e. $\left.\left\{H_{i}, H_{j}\right\}=0\right)$ and $m=2 n+k$. For most values of $c_{i} \in \mathbb{R}$, the invariant manifolds $\bigcap_{i=1}^{n+k}\left\{x \in \mathbb{R}^{m}: H_{i}=c_{i}\right\}$, are compact and connected. Then, according to the Arnold-Liouville theorem [5], there exists a diffeomorphism

$$
\Phi: \bigcap_{i=1}^{n+k}\left\{x \in \mathbb{R}^{m}: H_{i}=c_{i}\right\} \longrightarrow \mathbb{R}^{n} / \text { Lattice, }
$$

and the solutions of the system (5.1) are straight lines motions on these tori.

b) The invariant manifolds, thought of as affine varieties in $\mathbb{C}^{m}$ (non-compact) can be completed into complex algebraic tori, i.e.,

$$
\bigcap_{i=1}^{n+k}\left\{x \in \mathbb{C}^{m}: H_{i}=c_{i}\right\}=\mathbb{T}_{c}^{n} \backslash \text { a divisor } D \text { (i.e. codimension } 1 \text { subvarieties), }
$$

where the tori

$$
\mathbb{T}_{c}^{n}=\frac{\mathbb{C}^{n}}{\text { period Lattice }}=\text { complex algebraic torus (abelian variety) }
$$

depend on the $c^{\prime} s$.

Recall that algebraic means that $\mathbb{T}_{c}^{n}$ can be defined as an intersection $\bigcap_{i=1}^{M}\left\{\left(X_{0}, \ldots, X_{N}\right): P_{i}\left(X_{0}, \ldots, X_{N}\right)=0\right\}$, involving a large number of homogeneous polynomials $P_{i}$. The functions $x_{i}$ are required to be meromorphic on 
$\mathbb{T}_{c}^{n}$, and in particular in the natural coordinates $\left(t_{1}, \ldots, t_{n}\right)$ of $\mathbb{T}_{c}^{n}$ coming from $\mathbb{C}^{n}$, the functions $x_{i}=x_{i}\left(t_{1}, \ldots, t_{n}\right)$ are meromorphic and (5.1) defines straight line motion on $\mathbb{T}_{c}^{n}$. Condition $b$ ) means, in particular, there is an algebraic map $\left(x_{1}(t), \ldots, x_{m}(t)\right) \mapsto\left(s_{1}(t), \ldots, s_{n}(t)\right)$, making the following sums linear in $t$ :

$$
\sum_{i=1}^{n} \int_{s_{i}(0)}^{s_{i}(t)} \omega_{j}=\mu_{j} t, 1 \leq j \leq n, \mu_{j} \in \mathbb{C},
$$

where $\omega_{1}, \ldots, \omega_{n}$ denote holomorphic differentials on some algebraic curves.

Adler and van Moerbeke [2] have developed and used the following algebraic complete integrability criterion : If the hamiltonian system (5.1) is algebraic completely integrable, then each $x_{i}$ blows up for some value of $t \in \mathbb{C}$ and whenever it blows up, the solution $x(t)$ behaves as a Laurent series

$$
x_{i}=t^{-k_{i}}\left(x_{i}^{(0)}+x_{i}^{(1)} t+x_{i}^{(2)} t^{2}+\cdots\right), k_{i} \in \mathbb{Z}, \text { some } k_{i}>0,
$$

which admits $\operatorname{dim}$ (phase space) $-1=m-1$ free parameters. To explain the criterion, if the hamiltonian flow (5.1) is algebraic completely integrable, it means that the variables $x_{i}$ are meromorphic on the torus $\mathbb{T}_{c}^{n}$ and by compactness they must blow up along a codimension one subvariety (a divisor) $D \subset \mathbb{T}_{c}^{n}$. By the algebraic complete integrability definition, the flow (5.1) is a straight line motion in $\mathbb{T}_{c}^{n}$ and thus it must hit the divisor $D$ in at least one place. Moreover through every point of $D$, there is a straight line motion and therefore a Laurent expansion around that point of intersection. Hence the differential equation must admit Laurent expansions which depend on the $n-1$ parameters defining $D$ and the $n+k$ constants $c_{i}$ defining the torus $\mathbb{T}_{c}^{n}$, the total count is therefore $m-1=\operatorname{dim}($ phase space $)-1$ parameters.

The system (2.2) can be written in the form (5.1) with $m=4, n=2, k=0$

$$
\dot{x}=J \frac{\partial H}{\partial x} \equiv f(x) \quad x=\left(y_{1}, y_{2}, x_{1}, x_{2}\right)^{\top},
$$

$H=H_{1}(2)$ with $a_{3}=a_{4}=1, \frac{\partial H}{\partial x}=\left(\frac{\partial H}{\partial y_{1}}, \frac{\partial H}{\partial y_{2}}, \frac{\partial H}{\partial x_{1}}, \frac{\partial H}{\partial x_{2}}\right)^{\top}$ and $J=\left(\begin{array}{ll}0 & I \\ -I & 0\end{array}\right)$ a skew-symmetric matrix where $I$ is the $2 \times 2$ unit matrix and $O$ the $2 \times 2$ zero matrix. The second flow commuting with the first is regulated by the system

$$
\dot{x}=J \frac{\partial H_{2}}{\partial x}, x=\left(y_{1}, y_{2}, x_{1}, x_{2}\right)^{\top},
$$

with $H_{2}$ defined by (3.4). The main problem will be to complete the affine surface $V_{c}$ (3.5) into an abelian surface. A naive guess would be to take the natural compactification $\bar{V}_{c}=\bigcap_{i=1}^{2}\left\{X \in \mathbb{C P}^{4}: H_{i}(X)=c_{i} X_{0}^{2}\right\}$ of $V_{c}$ by projectivizing the equations. Indeed, this can never work for a general reason: an abelian variety $\widetilde{V}_{c}$ of dimension bigger or equal than two is never a complete intersection, that is it can never be descriped in some projective space $\mathbb{C P}^{n}$ by $n$-dim $\widetilde{V}_{c}$ global polynomial homogeneous equations. In other words, if $V_{c}(4.1)$ is to be the affine part of an abelian surface, $\bar{V}_{c}$ must have a singularity somewhere along the locus at infinity $\bar{V}_{c} \cap\left\{X_{0}=0\right\}$. An explicit calculation using the adjunction formula $[18,31]$ shows that we have :

Theorem 5.1. Let $\bar{V}_{c}$ be the projective variety on $\mathbb{C P}^{4}$ defined by

$$
\begin{gathered}
\frac{1}{2}\left(X_{1}^{2}+X_{2}^{2}+a_{1} Y_{1}^{2}+a_{2} Y_{2}^{2}\right) X_{0}^{2}+\frac{1}{4}\left(Y_{1}^{2}+Y_{2}^{2}\right)^{2}=c_{1} X_{0}^{4}, \\
\frac{1}{4}\left(a_{2} Y_{1}^{4}+a_{1} Y_{2}^{4}+\left(a_{1}+a_{2}\right) Y_{1}^{2} Y_{2}^{2}\right)+\frac{1}{4}\left(X_{1} Y_{2}-X_{2} Y_{1}\right)^{2} \\
\quad+\frac{1}{2}\left(a_{2} X_{1}^{2}+a_{1} X_{2}^{2}\right) X_{0}^{2}+\frac{1}{2} a_{1} a_{2}\left(Y_{1}^{2}+Y_{2}^{2}\right) X_{0}^{2}=c_{2} X_{0}^{4} .
\end{gathered}
$$

Then $\bar{V}_{c}$ is not an abelian surface. The variety $\bar{V}_{c}$ intersects the hyperplane at infinity $X_{0}=0$ according to two lines

$$
d_{\varepsilon}=\left(X_{0}, X_{1}, X_{2}, Y_{1}, Y_{2}\right)=\left(0, X_{1}, \varepsilon i X_{1}, Y_{1}, \varepsilon i Y_{1}\right), \varepsilon \equiv \pm 1,
$$

intersecting into one point $\left(0, X_{1}, X_{2}, 0,0\right)$ and $\bar{V}_{c}$ is singular all along $d_{\varepsilon}$.

The trajectories of the vector fields (5.2) hit every point of the singular locus at infinity and ignore the smooth locus at infinity. In fact, we shall show that the existence of meromorphic solutions to the differential equations (2.2) depending on 3 free parameters can be used to manufacture the tori, without ever going through the delicate procedure of blowing up and down. Information about the tori can then be gathered from the divisor. 
More precisely, we shall show that around the points of hitting, the system of differential equations (5.2) admit a Laurent expansion solution depending on 3 free parameters and in order to regularize the flow at infinity, we use these parameters to blowing up the variety $\bar{V}_{c}$ along the lines $d_{\varepsilon}$ (5.4). The new complex surface obtained in this fashion is compact, smooth and has two commuting vector fields on it; it is therefore an abelian surface and the two lines $d_{\varepsilon}$ turn into two copies of a smooth genus 3 hyperelliptic curves, intersecting doubly into two different points.

\section{Laurent series solutions and algebraic curves}

Consider points at infinity which are limit points of trajectories of the flow. In fact, there is a Laurent decomposition of such asymptotic solutions,

$$
\begin{aligned}
x(t) & \equiv\left(y_{1}(t), y_{2}(t), x_{1}(t), x_{2}(t)\right), \\
y_{1} & =\frac{y_{1}^{(0)}}{t}+y_{1}^{(1)}+y_{1}^{(2)} t+y_{1}^{(3)} t^{2}+y_{1}^{(4)} t^{3}+\cdots, \\
y_{2} & =\frac{y_{2}^{(0)}}{t}+y_{2}^{(1)}+y_{2}^{(2)} t+y_{2}^{(3)} t^{2}+y_{2}^{(4)} t^{3}+\cdots,
\end{aligned}
$$

$x_{1}=\dot{y}_{1}, x_{2}=\dot{y}_{2}$, and which depend on $\operatorname{dim}$ (phase space) $-1=3$ free parameters. Putting (6.1) into (5.2), solving inductively for the $x^{(k)}$, one finds at the $0^{\text {th }}$ step a non-linear equation,

$$
x^{(0)}+f\left(x^{(0)}\right)=0,
$$

and at the $k^{t h}$ step, a linear system of equations,

$$
(\mathrm{J}-k \mathrm{I}) x^{(k)}=\left\{\begin{array}{c}
0 \text { for } k=1 \\
\text { quadratic polynomial in } x^{(1)}, \ldots, x^{(k-1)} \text { for } k>1
\end{array},\right.
$$

where J denotes the Jacobian map of (6.2). One parameter $\alpha$ appear at the $0^{\text {th }}$ step, i.e., in the resolution of (6.2) and the two remaining ones $\beta, \gamma$, at the $k^{t h}$ step, $k=3, k=4$ i.e., in the resolution of (6.3). These Laurent series solutions are explicitly given by

$$
\begin{aligned}
& y_{1}=\frac{\alpha}{t}+\frac{1}{6}\left(-a_{2} \alpha^{2}+\alpha^{2} a_{1}-2 a_{2}+3 a_{1}\right) \alpha t+\beta t^{2}+y_{1}^{(4)} t^{3}+\cdots \\
& y_{2}=\frac{\varepsilon i \sqrt{2+\alpha^{2}}}{t}+\frac{\varepsilon i}{6}\left(-a_{2} \alpha^{2}+a_{2}+\alpha^{2} a_{1}\right) \sqrt{2+\alpha^{2}} t+\frac{\varepsilon i \alpha \beta}{\sqrt{2+\alpha^{2}}} t^{2}+\gamma t^{3}+\cdots \\
& x_{1}=-\frac{\alpha}{t^{2}}+\frac{1}{6}\left(-a_{2} \alpha^{2}+\alpha^{2} a_{1}-2 a_{2}+3 a_{1}\right) \alpha+2 \beta t+3 y_{1}^{(4)} t^{2}+\cdots \\
& x_{2}=-\frac{\varepsilon i \sqrt{2+\alpha^{2}}}{t^{2}}+\frac{\varepsilon i}{6}\left(-a_{2} \alpha^{2}+a_{2}+\alpha^{2} a_{1}\right) \sqrt{2+\alpha^{2}}+\frac{2 \varepsilon i \alpha \beta}{\sqrt{2+\alpha^{2}}} t+3 \gamma t^{2}+\cdots
\end{aligned}
$$

with $y_{1}^{(4)}$ given by

$$
\begin{gathered}
y_{1}^{(4)}=-\frac{\alpha}{24\left(2+\alpha^{2}\right)}\left(2 a_{2}^{2}-8 a_{2} a_{1}+6 a_{1}^{2}+2 \alpha^{4} a_{1}^{2}+2 a_{2}^{2} \alpha^{4}+5 a_{2}^{2} \alpha^{2}-12 a_{1} a_{2} \alpha^{2}\right. \\
\left.+7 \alpha^{2} a_{1}^{2}+24 \varepsilon i \sqrt{\left(2+\alpha^{2}\right)} \gamma-4 a_{2} \alpha^{4} a_{1}\right),
\end{gathered}
$$

and $\varepsilon= \pm 1$. Using the majorant method, we can show that the formal Laurent series solution are convergent. We have

Theorem 6.1. a) The non identically zero solutions of (6.2) define a sphere. The system (6.3) has 1 degree of freedom for $k=3$ and $k=4$.

$b$ ) The differential equations (5.2) admit Laurent series solutions depending on 3 free parameters $\alpha, \beta$ and $\gamma$. The first free parameter $\alpha$ appears in the resolution of (6.2) and the two remaining ones $\beta, \gamma$ at the $k^{\text {th }}$ step, $k=3, k=4$. These Laurent series solutions are explicitly given above. 
Now, taking into account only solutions trajectories lying on the surface $V_{c}$ (3.5), we show that the Laurent solutions are parameterized by two copies $C_{\varepsilon}$ of the same hyperelliptic curve $C$ for $\varepsilon= \pm 1$. To be precise we search for the set of Laurent solutions which remain confined to a fixed affine invariant surface, related to specific values of $c_{1}, c_{2}$, i.e.,

$$
\begin{aligned}
& C=\text { closure of the continuous components of } \\
& \text { \{Laurent series solutions } \left.x(t) \text { such that } H_{i}(x(t))=c_{i}, 1 \leq i \leq 2\right\} \text {, } \\
& =\bigcap_{i=1}^{2}\left\{t^{0}-\text { coefficient of } H_{i}(x(t))=c_{i}\right\} \text {, } \\
& =\text { an algebraic curve : }
\end{aligned}
$$

$$
\beta^{2}=\left(2+\alpha^{2}\right)\left(A_{1} \alpha^{6}+A_{2} \alpha^{4}+A_{3} \alpha^{2}+A_{4}\right),
$$

where $A_{1}=\frac{\left(a_{2}-a_{1}\right)^{3}}{72}, A_{2}=\frac{\left(a_{2}-a_{1}\right)^{2}\left(a_{2}-2 a_{1}\right)}{36}, A_{3}=\frac{\left(a_{2}-a_{1}\right)\left(a_{1}^{2}-a_{1} a_{2}-c_{1}\right)}{18}$ and $A_{4}=\frac{\left(a_{1} c_{1}-c_{2}\right)}{9}$. This is a eighth-order equation, so $C$ is an hyperelliptic curve of genus 3 and the Laurent solutions (6.4) restricted to the surface $V_{c}$ (3.5) are parameterized by two copies $C_{+1}$ and $C_{-1}$ of the same hyperelliptic curve $C$. Moreover, the map

$$
\sigma: C \longrightarrow C,(\alpha, \beta) \longmapsto(-\alpha, \beta),
$$

is an involution on $C$ and the quotient $C_{0}=C / \sigma$ is an elliptic curve :

$$
C_{0}: \beta^{2}=(2+\zeta)\left(A_{1} \zeta^{3}+A_{2} \zeta^{2}+A_{3} \zeta+A_{4}\right) .
$$

The curve $C$ is a double ramified covering of $C_{0}$,

$$
\varphi: C \longrightarrow C_{0},(\alpha, \beta) \longmapsto(\zeta, \beta),
$$

ramified at the four points covering $\zeta=0$ and $\infty$. The curve $C$ can also be seen as a 2 -sheeted unramified cover

$$
\pi: C \longrightarrow \mathcal{H},(\alpha, \beta) \longmapsto(\zeta, \eta)
$$

of the following hyperelliptic curve $\mathcal{H}$ of genus 2 :

$$
\mathcal{H}: \eta^{2}=\zeta(2+\zeta)\left(A_{1} \zeta^{3}+A_{2} \zeta^{2}+A_{3} \zeta+A_{4}\right) .
$$

Thus, we have proved

Theorem 6.2. The Laurent solutions (6.4) restricted to the surface $V_{c}$ (3.5) are parameterized by two copies $C_{+1}$ and $C_{-1}$ of the same hyperelliptic curve $C$ (6.5) of genus 3. The latter is a double ramified cover of an elliptic curve $C_{0}(6.7)$ and a 2-1 unramified cover of an hyperelliptic curve $\mathcal{H}$ (6.10) of genus 2.

\section{Affine parts of abelian surfaces as complete intersection of two quartics}

We recall some various useful facts about abelian varieties which will be needed (details can be found in [18, 31]). Let $\widetilde{V}_{c}$ be a smooth surface compactifying $V_{c}$ (3.5) and $D$ the embedding of $C_{+1}+C_{-1}$ into $\mathbb{C P}^{N}$. Consider a basis $1, f_{1}, \ldots, f_{N}$ of the vector space $\mathcal{L}(D)$ of meromorphic functions on $\widetilde{V}_{c}$ with at worst a simple pole along $D$ and the map $\widetilde{V}_{c} \longrightarrow \mathbb{C P}^{N}, p \longmapsto\left[1, f_{1}(p), \ldots, f_{N}(p)\right]$, considered projectively, because if at $p$ some $f_{i}(p)=\infty$, we divide by $f_{i}$ having the highest order pole near $p$, which makes every element finite. The Kodaira embedding theorem tells us that if the line bundle associated with the divisor is positive, then for $k \in \mathbb{N}$, the functions of $\mathcal{L}(k D)$ embed smoothly $\widetilde{V}_{c}$ into $\mathbb{C P}^{N}$ and then by Chow's theorem, $\widetilde{V}_{c}=\bigcap_{i}\left\{X \in \mathbb{C P}^{N}: P_{i}(X)=0\right\}$, where $P_{i}(X)$ are homogeneous polynomials, i.e., $\widetilde{V}_{c}$ can be realized as an algebraic variety. In fact in our case, $k=2$ suffices, i.e., the divisor $2 D$ provides a smooth embedding into $\mathbb{C P}^{15}$, via the meromorphic sections of $L(2 D)$. Put $S \equiv 2 D$ and let

$$
\chi(S)=\operatorname{dim} H^{0}\left(\widetilde{V}_{c}, \mathcal{O}_{\widetilde{V}_{c}}(S)\right)-\operatorname{dim} H^{1}\left(\widetilde{V}_{c}, \mathcal{O}_{\widetilde{V}_{c}}(S)\right),
$$


be the Euler characteristic of $S$. The adjunction formula and the Riemann-Roch theorem for divisors on $\widetilde{V}_{c}$ imply that

$$
g(S)=\frac{K_{\widetilde{V}_{c}} \cdot S+S . S}{2}+1, \quad \chi(S)=p_{a}\left(\widetilde{V}_{c}\right)+1+\frac{1}{2}\left(S \cdot\left(S-K_{\widetilde{V}_{c}}\right)\right),
$$

$g(S)$ is the geometric genus of $S, p_{a}\left(\widetilde{V}_{c}\right)$ is the arithmetic genus of $\widetilde{V}_{c}$ and $S . S$ denote the number of intersection points of $S$ with $a+S$ where $a+S$ is a small translation by $a$ of $S$ on $\widetilde{V}_{c}$. Using Kodaira-Serre duality:

$$
\begin{aligned}
\chi(S) & =\operatorname{dim} \mathcal{L}(S)-\operatorname{dim} H^{1}\left(\widetilde{V}_{c}, \Omega^{2}\left(S \otimes \mathcal{O}_{\widetilde{V}_{c}}(-S)\right)\right), \\
& =\operatorname{dim} \mathcal{L}(S)-\operatorname{dim} H^{1}\left(\widetilde{V}_{c}, \mathcal{O}_{\widetilde{V}_{c}}\left(K_{\widetilde{V}_{c}} \backslash S\right)\right),
\end{aligned}
$$

Kodaira-Nakano vanishing theorem $: \operatorname{dim} H^{1}\left(\widetilde{V}_{c}, \mathcal{O}_{\widetilde{V}_{c}}(S)\right)=0$, then it is easy to see that if $\widetilde{V}_{c}$ is an abelian surface (i.e., $K_{\widetilde{V}_{c}}=0, p_{a}\left(\widetilde{V}_{c}\right)=-1$ ),

$$
g(S)-1=\frac{S . S}{2}=\chi(S)=\operatorname{dim} \mathcal{L}(S)\left(\equiv h^{0}(\mathcal{L})\right)=N+1 .
$$

Let $\delta_{1}, \delta_{2} \in \mathbb{N}^{*}, \delta_{1} \mid \delta_{2}$, be the elementary divisors. Recall that a polarization of type $\left(\delta_{1}, \delta_{2}\right)$ on $\widetilde{V}_{c}$ is defined by an ample line bundle $\mathcal{L}$ on $\widetilde{V}_{c}$, such that the group of translation $t$ on $\widetilde{V}_{c}$ with $t^{*} \mathcal{L} \simeq \mathcal{L}$ is isomorphic with $\left(\mathbb{Z}_{\delta_{1}} \times \mathbb{Z}_{\delta_{2}}\right)^{2}$. The integers $\delta_{1}$ and $\delta_{2}$ are related as follows :

$$
\operatorname{dim} \mathcal{L}(S)=\delta_{1} \delta_{2}
$$

Theorem 7.1. a) The 16 functions $1, f_{1}, \ldots, f_{15}$ defined in (7.4) provide the embedding of $\widetilde{V}_{c}$ in projective space $\mathbb{C P}^{15}$. The divisor $D=C_{+1}+C_{-1}$ obtained has genus 5 and $S \equiv 2 D$ has genus 17 .

b) The orbits of the vector field (5.2) running through $S$ form a smooth surface $\Sigma$ near $S$ such that $\Sigma \backslash S \subseteq \widetilde{V}_{c}$. Moreover, the variety $\widetilde{V}_{c}=V_{c} \bigcup \Sigma$ is smooth, compact and connected.

c) The variety $\widetilde{V}_{c}$ comes equipped with two everywhere independent commuting vector fields, which extend holomorphically on $\widetilde{V}_{c}$. The flow (5.2) evolves on abelian surface $\widetilde{V}_{c}=\mathbb{T}_{c}^{2} \subset \mathbb{C P}^{15}$ with the polarization of type $(2,2)$ and it will be expressed in terms of abelian integrals, involving the differentials (7.5).

Proof. a) Based on the motivation above, it is easy to find a set of polynomial functions $\left\{1, f_{1}, \ldots, f_{N}\right\}$ in $\mathcal{L}(S)$, i.e., $\mathcal{L}(2 D)$ such that the embedding of $S$ with those functions into $\mathbb{C P}^{N}$ yields a curve of genus $N+2$. Straightforward calculation, using asymptotic expansions, shows that the space $\mathcal{L}(S)$ is spanned by the following functions

$$
\mathcal{L}(S)=\left\{1, f_{1}, \ldots, f_{15}\right\}
$$

where $f_{1}=y_{1}, f_{2}=y_{2}, f_{3}=x_{1}, f_{4}=x_{2}, f_{5}=f_{1}^{2}, f_{6}=f_{2}^{2}, f_{7}=f_{1} f_{2}, f_{8}=f_{1} f_{4}-f_{2} f_{3}, f_{9}=f_{1} f_{8}=, f_{10}=f_{2} f_{8}$, $f_{11}=f_{8}^{2}, f_{12}=\left(f_{5}+f_{6}\right) f_{8}+2\left(a_{1} f_{1} f_{4}-a_{2} f_{2} f_{3}\right), f_{13}=\left(f_{5}+f_{6}\right) f_{1} f_{2}+2 f_{3} f_{4}, f_{14}=f_{3} f_{8}+\left(a_{2}-a_{1}\right) f_{1} f_{7}, f_{15}=$ $f_{4} f_{8}+\left(a_{2}-a_{1}\right) f_{2} f_{7}$. Using these functions $1, f_{1}, \ldots, f_{15}$, one embeds each of the curves $C_{+1}$ and $C_{-1}$ into $\mathbb{C P}^{15}$. Let us look more closely at certain points of interest on the curve $C$. In a neighborhood of the point $s_{k} \equiv(\alpha= \pm i \sqrt{2}, \beta=0), k=1,2$, it is convenient to divide the vector in (7.2) evaluated on $C$, by $f_{12}$. Then, upon putting

we find

$$
\left(\frac{1}{f_{12}}, \frac{f_{1}}{f_{12}}, \ldots, \frac{f_{15}}{f_{12}}\right) \equiv F_{k}^{(0)}+F_{k}^{(1)} t+\cdots, 0 \leq k \leq 15,
$$

$$
F_{k}^{(0)}\left(s_{k}\right)=\left(0,0,0, \frac{\mp \sqrt{2}}{4 \varepsilon \sqrt{a_{2} c_{1}-c_{2}}}, 0, \frac{ \pm i}{2 \varepsilon \sqrt{a_{2} c_{1}-c_{2}}}, 0,0,0,0,0,0,1,0, \frac{\mp i \sqrt{2}}{2}, 0\right),
$$

which leads to eight different points in $\mathbb{C P}^{15}$ according to the sign of $\varepsilon= \pm 1$. Using the transformation $C_{+1} \rightarrow C_{-1}:(\alpha, \beta) \mapsto(-\alpha, \beta)$, we have $\left.F_{k}^{(0)}\left(s_{k}\right)\right|_{C_{+1}}=\left.F_{k}^{(0)}\left(s_{k}\right)\right|_{C_{-1}}$, implying that the two points $s_{k}$ on one curve are identified pairwise with the two corresponding points on the other curve. Also, we have

$$
\left.\frac{\partial F_{k}^{(0)}\left(s_{k}\right)}{\partial \alpha}\right|_{C_{+1}}=\left.\frac{\partial F_{k}^{(0)}\left(s_{k}\right)}{\partial \alpha}\right|_{C_{-1}}
$$


then the curves $C_{+1}$ and $C_{-1}$ have two points in common $s_{1} \equiv(\alpha=i \sqrt{2}, \beta=0)$ and $s_{2} \equiv(\alpha=-i \sqrt{2}, \beta=0)$, at which they are tangent to each other. In a neighborhood of the point $\left(\alpha=0, \beta= \pm \frac{\sqrt{2}}{3} \sqrt{a_{1} c_{1}-c_{2}}\right)$, dividing the vector in (7.2) by $f_{12}$ and taking the limit when $\alpha$ tends to 0 , we find the following four points in $\mathbb{C P}^{15}$ :

$$
\left(0,0,0,0, \mp \frac{3 \sqrt{2}}{\sqrt{a_{1} c_{1}-c_{2}}}, 0, \pm \frac{i}{2 \varepsilon \sqrt{a_{1} c_{1}-c_{2}}}, 0,0,0,0,0,1,0,0,-\frac{\sqrt{2}}{2 i \varepsilon}\right), \varepsilon= \pm 1
$$

Also, in a neighborhood of the point $\alpha=\infty$, dividing the vector in (7.2) by $f_{11}$ and letting $\alpha \nearrow \infty$, we find

$$
F_{k}^{(0)}(\alpha \nearrow \infty)=\left(0, \ldots, 1,0,-\frac{i \varepsilon}{a_{2}-a_{1}}, \mp \frac{i \varepsilon \sqrt{2}}{2 \sqrt{a_{2}-a_{1}}}, \pm \frac{\sqrt{2}}{2 \sqrt{a_{2}-a_{1}}}\right),
$$

which leads to four different points according to the sign of $\varepsilon= \pm 1$. The divisor $D=C_{+1}+C_{-1}$ obtained in this way has genus 5 and thus $S=2 D$ has genus 17 , satisfying the requirement (7.1).

b) Let $\psi(t, p)=\left\{x(t)=\left(y_{1}(t), y_{2}(t), x_{1}(t), x_{2}(t)\right): t \in \mathbb{C}, 0\langle|t|\langle\varepsilon\}\right.$, be the orbit of the vector field (5.2) going through the point $p \in S$. Let $\Sigma_{p} \subset \mathbb{C P}^{15}$ be the surface element formed by the divisor $S$ and the orbits going through $p$, and set $\Sigma \equiv \bigcup_{p \in S} \Sigma_{p}$. Consider the curve $S^{\prime}=\mathcal{H} \cap \Sigma$ where $\mathcal{H} \subset \mathbb{C P}^{15}$ is a hyperplane transversal to the direction of the flow. If $S^{\prime}$ is smooth, then using the implicit function theorem the surface $\Sigma$ is smooth. But if $S^{\prime}$ is singular at 0 , then $\Sigma$ would be singular along the trajectory ( $t$-axis) which go immediately into the affine part $V_{c}$. Hence, $V_{c}$ would be singular which is a contradiction because $V_{c}$ is the fibre of a morphism from $\mathbb{C}^{4}$ to $\mathbb{C}^{2}$ and so smooth for almost all the two constants of the motion $c_{i}$. Next, let $\bar{V}_{c}$ be the projective closure of $V_{c}$ into $\mathbb{C P}^{4}$, let $X=\left(X_{0}, X_{1}, X_{2}, Y_{1}, Y_{2}\right) \in \mathbb{C P}^{4}$ and let $I=\bar{V}_{c} \bigcap\left\{X_{0}=0\right\}$ be the locus at infinity (see theorem 4). Consider the map $\bar{V}_{c} \subseteq \mathbb{C P}^{4} \rightarrow \mathbb{C P}^{15}, X \mapsto f(X)$, where $f=\left(1, f_{1}, \ldots, f_{15}\right) \in L(S)$ and let $\widetilde{V}_{c}=f\left(\bar{V}_{c}\right)$. In a neighborhood $V(p) \subseteq \mathbb{C P}^{15}$ of $p$, we have $\Sigma_{p}=\widetilde{V}_{c}$ and $\Sigma_{p} \backslash S \subseteq V_{c}$. Otherwise there would exist an element of surface $\Sigma_{p}^{\prime} \subseteq \widetilde{V}_{c}$ such that $\Sigma_{p} \cap \Sigma_{p}^{\prime}=t$-axis, orbit $\psi(t, p)=t$-axis $\backslash p \subseteq V_{c}$ and hence $V_{c}$ would be singular along the $t$-axis which is impossible. Since the variety $\bar{V}_{c} \cap\left\{X_{0} \neq 0\right\}$ is irreducible and since the generic hyperplane section $\mathcal{H}_{g e n}$. of $\bar{V}_{c}$ is also irreducible, all hyperplane sections are connected and hence $I$ is also connected. Now, consider the graph $\Gamma_{f} \subseteq \mathbb{C P}^{4} \times \mathbb{C P}^{15}$ of the map $f$, which is irreducible together with $\bar{V}_{c}$. It follows from the irreducibility of $I$ that a generic hyperplane section $\Gamma_{f} \cap\left\{\mathcal{H}_{\text {gen. }} \times \mathbb{C P}^{15}\right\}$ is irreducible, hence the special hyperplane section $\Gamma_{f} \cap\left\{\left\{X_{0}=0\right\} \times \mathbb{C P}^{15}\right\}$ is connected and therefore the projection map $\operatorname{proj}_{\mathbb{C P}^{15}}\left[\Gamma_{f} \cap\left\{\left\{X_{0}=0\right\} \times \mathbb{C P}^{15}\right]=f(I) \equiv S\right.$, is connected. Hence, the variety $V_{c} \cup \Sigma=\widetilde{V}_{c}$ is compact, connected and embeds smoothly into $\mathbb{C P}^{15}$ via $f$.

c) Let $t_{1}$ and $t_{2}$ be the time variables respectively of the vector field $X_{1}: \dot{x}=J \frac{\partial H_{1}}{\partial x}=\frac{\partial x}{\partial t_{1}}(13)$ and the second vector field $X_{2}: \dot{x}=J \frac{\partial H_{2}}{\partial x}=\frac{\partial x}{\partial t_{2}}$ commuting with the first one as well. Let $g^{t_{1}}$ and $g^{t_{2}}$ be the flows generated respectively by the first and second vector fields respectively. For $p \in S$ and for small $\varepsilon>0, g^{t_{1}}(p)$, $\forall t_{1} \in \mathbb{C}, 0<\left|t_{1}\right|<\varepsilon$, is well defined and $g^{t_{1}}(p) \in V_{c}$. We define $g^{t_{2}}$ on $V_{c}$ by $g^{t_{2}}(q)=g^{-t_{1}} g^{t_{2}} g^{t_{1}}(q), q \in U(p)=$ $g^{-t_{1}}\left(U\left(g^{t_{1}}(p)\right)\right)$, where $U(p)$ is a neighborhood of $p$. By commutativity one can see that $g^{t_{2}}$ is independent of $t_{1}$; $g^{-t_{1}-\varepsilon_{1}} g^{t_{2}} g^{t_{1}+\varepsilon_{1}}(q)=g^{-t_{1}} g^{-\varepsilon_{1}} g^{t_{2}} g^{t_{1}} g^{\varepsilon_{1}}=g^{-t_{1}} g^{t_{2}} g^{t_{1}}(q)$. We affirm that $g^{t_{2}}(q)$ is holomorphic away from $S$. This because $g^{t_{2}} g^{t_{1}}(q)$ is holomorphic away from $S$ and that $g^{t_{1}}$ is holomorphic in $U(p)$ and maps bi-holomorphically $U(p)$ onto $U\left(g^{t_{1}}(p)\right)$. Since the flows $g^{t_{1}}$ and $g^{t_{2}}$ are holomorphic and independent on $S$, we can show along the same lines as in the Arnold-Liouville theorem [5] that $\widetilde{V}_{c}$ is a torus. And that will done, by considering the holomorphic map $\mathbb{C}^{2} \longrightarrow \widetilde{V}_{c}\left(t_{1}, t_{2}\right) \longmapsto g^{t_{1}} g^{t_{2}}(p)$, for a fixed origin $p \in V_{c}$. Then lattice $=\left\{\left(t_{1}, t_{2}\right) \in \mathbb{C}^{2}\right.$ : $\left.g^{t_{1}} g^{t_{2}}(p)=p\right\}$, is a lattice of $\mathbb{C}^{2}$ and hence $\mathbb{T}_{c}^{2}=\mathbb{C}^{2} /$ lattice $\rightarrow \widetilde{V}_{c}$ is a biholomorphic diffeomorphism. Therefore $\widetilde{V}_{c} \subseteq \mathbb{C P}^{15}$ is conformal to a complex torus $\mathbb{T}_{c}^{2}$ and an abelian surface as a consequence of Chow theorem. We now prove that the divisor $S \equiv 2 D$ defines on the abelian surface $\widetilde{V}_{c}$ a polarization either of type $(2,8)$. Note that the affine invariant surface $V_{c}$, has the following involution $\sigma:\left(y_{1}, y_{2}, x_{1}, x_{2}\right) \mapsto\left(y_{1},-y_{2},-x_{1}, x_{2}\right)$, which amounts to a reflection about some appropriately chosen origin on $V_{c}$. This map acts on the parameters of the Laurent solution (19) as follows $(t, \alpha, \beta) \mapsto(-t,-\alpha, \beta)$. Since $\mathcal{L}$ is symmetric $\left(\sigma^{*} \mathcal{L} \simeq \mathcal{L}\right), \sigma$ can be lifted to $\mathcal{L}$ as an involution $\widetilde{\sigma}$ in two ways differing in sign and for each section (theta-function) $s \in H^{0}(\mathcal{L})$, we therefore have $\widetilde{\sigma} s= \pm s$. Recall that a section $s \in H^{0}(\mathcal{L})$ is called even (resp. odd) if $\widetilde{\sigma} s=+s($ resp. $\widetilde{\sigma} s=-s)$. Under $\widetilde{\sigma}$ the vector space $H^{0}(\mathcal{L})$ splits into an even and odd subspace $H^{0}(\mathcal{L})=H^{0}(\mathcal{L})^{\text {even }} \oplus H^{0}(\mathcal{L})^{\text {odd }}$, with $H^{0}(\mathcal{L})^{\text {even }}$ containing all the even sections and $H^{0}(\mathcal{L})^{\text {odd }}$ all odd ones. Using the inverse formula [35, p.331], we see after a small 
computation that

$$
\begin{aligned}
h^{0}(\mathcal{L})^{\text {even }} & \equiv \operatorname{dim} H^{0}(\mathcal{L})^{\text {even }}=\frac{\delta_{1} \delta_{2}}{2}+2^{-1+\sharp \text { even } \delta_{k}}, k=1,2 \\
h^{0}(\mathcal{L})^{\text {odd }} & \equiv \operatorname{dim} H^{0}(\mathcal{L})^{\text {odd }}=\frac{\delta_{1} \delta_{2}}{2}-2^{-1+\sharp \text { even } \delta_{k}}, k=1,2
\end{aligned}
$$

By the classification theory of ample line bundles on abelian varieties, we have $\widetilde{V}_{c} \simeq \mathbb{C}^{2} / L_{\Omega}$ with period lattice given by the columns of the matrix $\Omega=\left(\begin{array}{llll}\delta_{1} & 0 & a & c \\ 0 & \delta_{2} & c & b\end{array}\right), \operatorname{Im}\left(\begin{array}{ll}a & c \\ c & b\end{array}\right)>0$, according to (7.1) and (7.2) with $\delta_{1} \delta_{2}=h^{0}(\mathcal{L})=g(S)-1=16, \delta_{1} \mid \delta_{2}, \delta_{i} \in \mathbb{N}^{*}$. Hence we have three possibilities : (i) $\delta_{1}=1, \delta_{2}=16$. (ii) $\delta_{1}=2$, $\delta_{2}=8$, (iii) $\delta_{1}=4, \delta_{2}=4$. From formula (31), the corresponding line bundle $\mathcal{L}$ has in case $(i), 9$ even sections, 7 odd ones and in cases $(i i),($ iii $), 10$ even sections, 6 odd ones. Among the functions of $\mathcal{L}$, there are 10 even and 6 odd functions for the involution $\sigma$, showing that cases $(i i),($ iii $)$ are the only alternative and the period matrices have the form $\left(\begin{array}{ll}2 & 0 \\ 0 & 8\end{array} \mid Z\right), Z^{\top}=Z, \operatorname{Im} Z>0$ for the case $(i i)$ and $\left(\begin{array}{ll}4 & 0 \\ 0 & 4\end{array} \mid Z\right), Z^{\top}=Z, \operatorname{Im} Z>0$ for the case $(i i i)$. On the other hand, the squares $y_{1}^{2}, y_{2}^{2}$ are meromorphic on $J a c(\Gamma)$, whereas the coordinates $y_{1}, y_{2}$ themselves are meromorphic on a 4 -fold cover of $J a c(\Gamma)$. Hence $\widetilde{V}_{c}$ must be a 4 -fold cover of $J a c(\Gamma)$ obtained by doubling two of the four period vectors of $\operatorname{Jac}(\Gamma)$. As a result, $\widetilde{V}_{c}$ must have polarization $(2,2)$. To give a more explicit description of the periods of motion, observe that the variables $t_{1}$ and $t_{2}$ form a local system of coordinates on the torus $\widetilde{V}$ and consider their differentials $d t_{1}$ and $d t_{2}$ defined by $d t_{i}\left(X_{j}\right)=\delta_{i j}, 1 \leq i, j \leq 2$. Then we show, using the Laurent solutions, that the differentials $d t_{1}$ and $d t_{2}$ restricted to the curve $C$ (6.7), descend to two differentials on $C$ :

$$
\left.d t_{1}\right|_{C}=\frac{\alpha^{2} d \alpha}{\beta},=\omega_{1},\left.\quad d t_{2}\right|_{C}=\frac{d \alpha}{\beta}=\omega_{2}
$$

Clearly both $\omega_{1}$ and $\omega_{2}$ are holomorphic differentials. This concludes the proof of theorem 7.1.

\section{Additional note and Conclusion}

At the end of this paper, It is worth to mention some similar problems. Abelian varieties, very heavily studied by algebraic geometers, enjoy certain algebraic properties which can then be translated into differential equations and their Laurent solutions. Among the results presented in this paper, there is an explicit calculation of invariants for hamiltonian systems which cut out an open set in an abelian variety and various algebraic curves related to these systems are given explicitly. The integrable systems presented here are interesting problems, particular to experts of abelian varieties who may want to see explicit examples of a correspondence for varieties defined by different algebraic curves. The methods used are primarily analytical but heavily inspired by algebraic geometrical methods. Strange as it may seem, the use of the Lax spectral curve technique may not give the tori correctly, but perhaps with period doubling, in contrast with the statement that the correct tori would be obtained by the Kowalewski-Painlevé analysis. This indicated a need for caution in interpretation of the result for tori calculated from use of the Lax spectral curve technique. Moser [34] was aware of a similar situation in the context of the Jacobi's geodesic flow problem on ellipsoids. Another striking example of this phenomenon already appears in the Euler-equations associated to a class of geodesic flow on $S O(4)$ for a left-invariant diagonal metric. Haine [19] has given a proof using Kowalewski-Painlevé analysis that the four quadrics in this problem intersect in the affine part of an abelian surface characterized as the Prym variety $\operatorname{Prym}\left(C / C_{0}\right)$ where $C$ is a genus 3 curve and is a double covering of an elliptic curve $C_{0}$. In [1], Adler and van Moerbeke linearize the problem, using the method of isospectral deformations, on a 2dimensional Prym variety $\operatorname{Prym}\left(K / K_{0}\right)$ of another genus 3 curve over an elliptic curve $K_{0}$. However, the two abelian surfaces $\operatorname{Prym}\left(C / C_{0}\right)$ and $\operatorname{Prym}\left(K / K_{0}\right)$ are not ismorphic but only isogenous, they intimately related in the precise sense that they are dual of each other. It turns out that these two abelian surfaces are isomorphic only up to multiplication of some (not all) periods by 2. The natural coordinates are not meromorphic on the tori but only their squares, if one linearizes the problem via Lax spectral curve technique. In contrast these natural coordinates are meromorphic on the invariant surfaces and on their natural completion into tori using the Kowalewski-Painlevé analysis. The relation between the two sets of tori is as follows : one set can be obtained from the other by doubling some but not all periods. The relationship between the curves $C$ and $K$ 
is complicated : $\operatorname{Prym}\left(K / K_{0}\right) \backslash \Pi=\Theta \cap \operatorname{Prym}\left(K / K_{0}\right)=C$ where $\Theta$ is a translate of the Riemann's theta divisor on $\operatorname{Jac}(K)$ et $\Pi \subset \operatorname{Prym}\left(K / K_{0}\right)$ is a Zariski-open set and $\Theta \cap \operatorname{Prym}^{*}\left(K / K_{0}\right)=\Theta \cap \operatorname{Prym}\left(C / C_{0}\right)=K$ where $\Theta$ is a translate of the Riemann's theta divisor on $\operatorname{Jac}(C)$.

\section{References}

[1] Adler, M., van Moerbeke, P., Linearization of Hamiltonian systems, Jacobi varieties and representation theory. Adv. in Math. 38 (1980), 318-379.

[2] Adler, M., van Moerbeke, P., The complex geometry of the Kowalewski-Painlevé analysis. Invent. Math. 7 (1989), 3-51.

[3] Adler, M., van Moerbeke, P. and Vanhaecke, P., Algebraic integrability, Painlevé geometry and Lie algebras. A series of modern surveys in mathematics, Volume 47, Springer-Verlag, 2004.

[4] Airault. H., Mc Kean, H.P. and Moser, J., Rational and elliptic solutions of the KdV equation and a related many-body problem. Comm. Pure Appl. Math. 30 (1977), 94-148.

[5] Arnold, V.I., Mathematical methods in classical mechanics. Springer-Verlag, Berlin-Heidelberg- New York, 1978.

[6] Baker, S., Enolskii, V.Z. and Fordy, A.P., Integrable quartic potentials and coupled KdV equations. Phys. Lett. 201A (1995), 167-174.

[7] Barth, W., Abelian surfaces with (1,2)-polarization. Conf. on alg. geom., Sendai, 1985, Advanced studies in pure mathematics, 10 (1987), 41-84.

[8] Barth, W., Affine parts of abelian surfaces as complete intersections of four quadrics. Math. Ann. 278 (1987), 117-131.

[9] Belokolos, A.I. and Enol'skii, V.Z., Isospectral deformations of elliptic potentials. Russ. Math. Surveys, 44 (1989), 155-156.

[10] Belokolos, A.I., Bobenko, V.Z., Enol'skii, V.Z., Its, A.R. and Matveev, V.B., Algebro-Geometric approach to nonlinear integrable equations Springer-Verlag 1994.

[11] Bountis, T., Segur, H. and Vivaldi, F., Integrable hamiltonian systems and the Painlevé property. Phys. Rev. A25 (1982), 1257-1264.

[12] Christiansen, P.L., Eilbeck, J.C., Enolskii, V.Z. and Kostov, N.A., Quasi-periodic solutions of the coupled nonlinear Schrödinger equations. Proc. R. Soc. Lond. A451 (1995), 685-700.

[13] Conte, R., Musette, M. and Verhoeven, C., Completeness of the cubic and quartic Hénon-Heiles hamiltonians. Theor. Math. Phys. 144 (2005), 888-898.

[14] Eilbeck, J.C., Enolskii, V.Z., Kuznetsov, V.B. and Leykin, D.V., Linear r-matrix algebra for systems separable in parabolic coordinates. Phys. Lett. 180A (1993), 208-214.

[15] Eilbeck, J.C., Enolskii, V.Z., Kuznetsov, V.B. and Tsiganov, A.V., Linear r-matrix algebra for classical separable systems. J. Phys. A.: Math Gen. 27 (1994), 567-578

[16] Eilbeck, J.C., Enolskii, V.Z., Elliptic solutions and blow-up in an integrable Hénon-Heiles system. Proc. Roy. Soc. Edinburgh, A124 (1994), 1151-1164.

[17] Grammaticos, B. Dorozzi, B., Ramani, A., Integrability of hamiltonians with third and fourth-degree polynomial potentials. J. Math. Phys. 24 (1983), 2289-2295.

[18] Griffiths, P.A., Harris,J., Principles of algebraic geometry.Wiley-Interscience 1978.

[19] Haine, L., Geodesic flow on $S O(4)$ and Abelian surfaces. Math. Ann. 263 (1983), 435-472.

[20] Halphen, G. H., Mémoire sur la réduction des équations différentielles linéaires aux formes intégrales. Mémoires présentés par divers savants à l'Académie des sciences de l'Institut de France 28, 1-307, 1884.

[21] Hermite, C., Oeuvres de Charles Hermites. Vol. III, Paris, Gauthier-Villar, 1912.

[22] Hietarinta, J., Classical versus quantum integrability. J. Math. Phys. 25 (1984), 1833-1840.

[23] Hietarinta, J., Direct methods for the search of the second invariant. Phys. Rep. 147 (1987), 87-154.

[24] Kasperczuk, S., Integrability of the Yang-Mills hamiltonian system. Celes. Mech. and Dyn. Astr. 58 (1994), 387-391. Erratum Celes. Mech. and Dyn. Astr. 60 (1994), 289.

[25] Kostov, N.A., Quasi-periodical solutions of the integrable dynamical systems related to Hill's equation. Lett. Math. Phys. 17 (1989), $95-104$.

[26] Kowalewski, S., Sur le problème de la rotation d'un corps solide autour d'un point fixe. Acta Math. 12 (1889), 177-232.

[27] Lesfari, A., Abelian surfaces and Kowalewski's top. Ann. Scient. École Norm. Sup. Paris, $4^{e}$ série, t.21 (1988), 193-223.

[28] Lesfari, A., Prym varieties and applications. J. Geom. Phys., 58, 1063-1079 (2008).

[29] Lesfari, A., Integrable systems and complex geometry. Lobachevskii Journal of Mathematics, Vol.30, No.4, 292-326 (2009).

[30] Lesfari, A.: Algebraic integrability : the Adler-van Moerbeke approach. Regul. Chaotic Dyn. 16 (2011), Nos.3-4, pp.187-209.

[31] Lesfari, A., Introduction à la géométrie algébrique complexe. Hermann, Paris 2015.

[32] McKean, H.P. and van Moerbeke, P., The Spectrum of Hill's Equation. Invent. Math. 30 (1975), 217-274.

[33] Moser, J., Three integrable Hamiltonian systems connected with isospectral deformations. Adv. Math. 16 (1975), 197-219.

[34] Moser, J., Geometry of quadrics and spectral theory. Lecture delivred at the symposium in honor of S.S. Chern, Berkeley, 1979. Springer, Berlin, Heidelberg, New-York 1980.

[35] Mumford, D., On the equations defining abelian varieties I, II, III. Invent. Math. 1 (1966), 287-354, 3 (1967), 75-135, 3 (1967), $215-244$.

[36] Mumford, D., Tata lectures on theta I, II. Progress in mathematics. Birkhaüser, Boston, 1983.

[37] Novikov, S.P., The periodic problem for Korteweg-de Vries equation. Funct. Anal. Pril. 8 (1974), 53-66.

[38] Perelomov, A.M., Integrable systems of classical mechanics and Lie algebras. Birkhäuser Verlag 1990

[39] Ravoson, V., Ramani, A. and Grammaticos, B., Generalized separability for a hamiltonian with nonseparable quartic potential. Phys. Lett. 191A (1994), 91-95.

[40] Smirnov, A. O., Finite-gap elliptic solutions of the KdV equation. Acta Appl. Math. 36 (1994), 125-199.

[41] Tondo, G., On the integrability of stationary and restricted flows of the KdV hierarchy. J. Phys. A: Mat. Gen. 28 (1995), $5097-5115$.

[42] Vanhaecke, P., Linearising two-dimensional integrable systems and the construction of action-angle variables. Math. Z. 211 (1992), $265-313$.

[43] Vanhaecke, P., Integrable systems in the realm of algebraic geometry. Lecture Notes in Math. 1638, Springer-Verlag, 2001.

[44] van Moerbeke, P. and Mumford, D., The spectrum of difference operators and algebraic curves. Acta Math. 143 (1979), 93-154. 
[45] Verhoeven, C., Musette, M. and Conte, R., General solution of hamiltonians withs extend cubic and quartic potentials. Theor. Math. Phys. 134 (2003), 128-138.

[46] Wojciechowski, S., On a Lax-type representation and separability of the anisotropic harmonic oscillator in a radial quartic potential. Lett. Nuovo Cimento, 41 (1984), 361-369.

[47] Wojciechowski, S., Integrability of one particle in a perturbed central quartic potential. Physica Scripta, 31 (1985), $433-438$.

[48] Yoshida, H., Existence of exponentially unstable solutions and the non-integrability of homogeneous hamiltonian. Physica D, 21 (1986), 163-170.

[49] Zakharov, V.E., Manakov, S.V., Novikov, S.P. and Pitaevskii, L.P., Soliton theory, inverse scattering method. Moscow: Nauka, 1980.

[50] Ziglin, S.L., Branching of solutions and non-existence of first integrals in hamiltonian mechanics II. Funct. Anal. and its appl. 17 (1983), 6-17.

\section{Affiliations}

A. LESFARI

AdDress: Chouaib Doukkali University, Faculty of Sciences, Dept. of Mathematics, B.P. 20, 24000, El Jadida, Morocco.

E-MAIL: lesfariahmed@yahoo.fr

ORCID ID : orcid.org/0000-0001-6213-4301 